\title{
Performance Analysis of a Novel Swing Crank- slide Bar Beam Pumping Unit
}

\author{
Xiaoqing Kang ${ }^{\mathrm{a}}$, Fei Zhang, and Tao Ren \\ School of Mechanical Engineering, Xi'an Shiyou University, Shaanxi, Xi'an, 710065, P.R.China \\ axqkangw@126.com.
}

Keywords: Crank-slide bar beam pumping unit; Dynamic analysis; Energy saving.

\begin{abstract}
A novel swing crank-slide bar beam pumping unit composes of motor, reducer, crank, slider, guide rod, frame and wire rope. Up and down stroke is performed by slider moving along guide-rod guide apparatus resulting guide rod up and down movement against supporting point of pumping frame. Crank automatically adjusts torque according to the changes of hanging point loading when slider moves along guide rod. This maintains the net torque of brace axis always at its best condition. The net torque curve is very smooth and uneven loading factor CLF value approaches 1 . The novel swing crank-slide bar beam pumping unit dramatically decreases the rated power and effective power, which indicates that the novel pumping unit has more notable energy conservation effect and better dynamic performance.
\end{abstract}

\section{Introduction}

At present, the main oil field production equipment of the ground is the beam pumping unit in our country. The beam pumping unit has been widely applied in oil production, because of its simple structure, reliable use, convenient operation and maintenance, the long-term safe operation in the harsh natural environment and other advantages. But there are three main problems in the beam pumping unit[1]: (1) the load fluctuation is large; (2) the installed power of the motor is too high; (3) the energy consumption of the pumping unit is larger..

The transmission mechanism of the pumping unit is one of the main influent factors of the energy consumption level. It is necessary to develop a most suitable transmission system for the pumping condition. It not only can reduce the energy consumption of pumping unit, but also reduce the volatility of load in depth. So the effective output power of the motor will be greatly reduced, and work efficiency is improved. In this paper, a novel swing crank-slide bar beam pumping unit is one of the best transmission mechanism which is in line with the requirements of pumping unit. The net torque curve of new swing crank-slide bar beam pumping unit is very smooth, and uneven loading factor CLF value approaches 1 . The novel swing crank-slide bar beam pumping unit dramatically decreases the rated power and effective power, which indicates that the novel pumping unit has more notable energy conservation effect and better dynamic performance.

\section{Scheme design}

Schematic diagram of the integral structure of a novel swing crank-slide bar beam pumping unit is Fig. 1. The main features are that the crank shaper mechanism is composed of crank, slider, guide rod and frame. The crank is hinged with a slider, the guide bar on slider sliding guide rail, which can be sliding guide or rolling guide. One end of the guide rod is hinged on the frame, and the other end is connected with the horsehead. When the crank rotates, the slider moves along guide-rod guide apparatus, and result guide rod up and down movement against supporting point of pumping frame at the same time. The Horsehead is arranged at the front end of the guide, which drives wire rope, rope rod connector and sucker rod to do reciprocating movement up and down. 


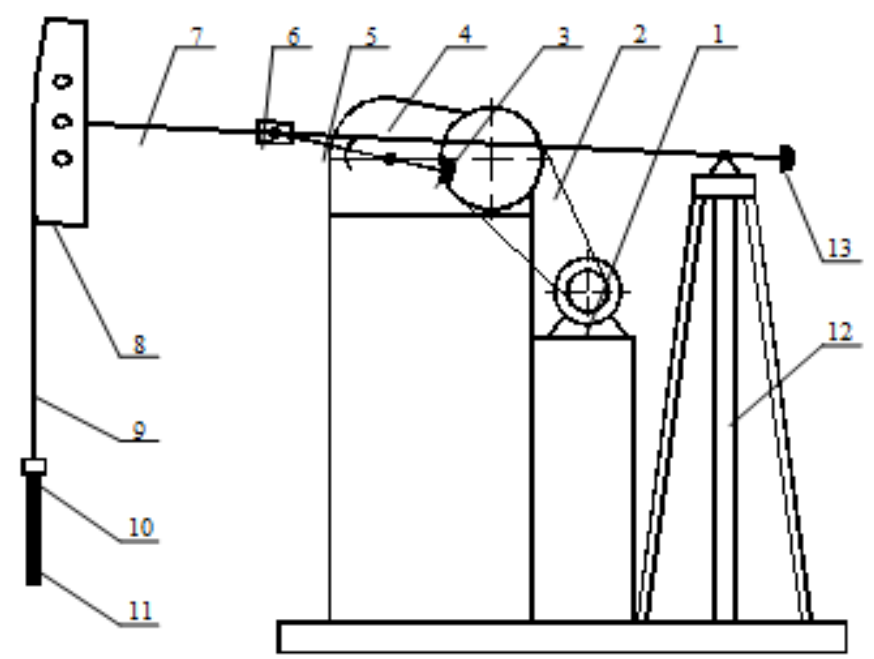

1 Motor; 2 Belt drive; 3 Crank counterbalance; 4 Reducer; 5 crank; 6 Slider; 7 Oscillating guard bar ; 8 Horsehead; 9 Wire rope; 10 Rope-rod connector; 11 Sucker rod; 12 Frame;

13 Balance weight of oscillating guard bar.

Fig.1 The schematic diagram of the integral structure of the swing crank-slide bar beam pumping unit

\section{Kinematic and dynamic analysis}

The motion and dynamic parameters of the pumping unit include[2]: the velocity and acceleration of the polished rod; the polished rod load, the net torque of brace axis, root mean square torque of brace axis, motor power.

Kinematic analysis. Kinematic diagram of a novel swing crank-slide bar beam pumping unit is Fig. 2 . The basic size of the mechanism is: the crank length is $R$. The length of the guide rod is $P$. The distance between the pivot point $B$ and the crank rotation center $O$ is $C$. The crank angle is $\theta$. The angle between the initial position of the crank angle and the frame is $\varphi$. The angle between the crank and the guide rod is $\alpha$, the angle between the guide rod and the frame is $\beta$.

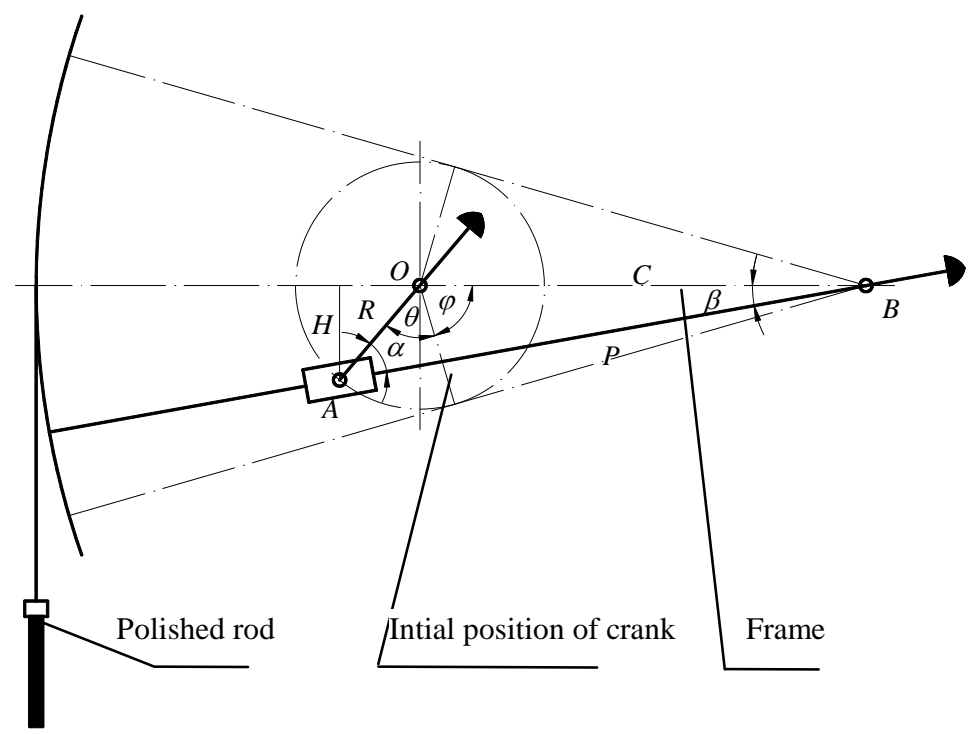

Fig.2 Kinematic diagram of the swing crank-slide bar beam pumping unit

(1) Angle between the initial position of the crank and the frame

$\phi=\arccos \left(\frac{R}{C}\right)$

(2) The maximum swing angle of the guide rod

$$
\beta_{\max }=\pi-2 \phi
$$


(3)The length of the line $\overline{A B}$

$\overline{A B}=\sqrt{R^{2}+C^{2}-2 R C \cos (\theta+\varphi)}$

(4)The length of the line $H$

$H=R \sin (\theta+\varphi)$

(5)The angle between the guide rod and the frame

$\beta=\arcsin \left(\frac{H}{\overline{A B}}\right)$

(6)The angle between the crank and the guide rod $\alpha=\pi-(\theta+\varphi)-\beta$

(7) The Maximum stroke of the pumping unit $S=P \times \beta_{\max }$

(8) The angular velocity of the guide bar

$$
\omega_{p}=\omega\left(1-\frac{C \cos \beta}{\overline{A B}}\right)
$$

Where, the angular velocity of brace axis is $\omega$.

(9) The velocity of the polished rod

$V=P \times \omega_{p}$

(10)The angular acceleration of the guide bar

$\varepsilon_{p}=\frac{d \omega_{p}}{d t}=\frac{\omega C}{\overline{A B}^{2}}\left(\overline{A B}(\cos \beta)^{\prime}-\overline{A B}^{\prime} \cos \beta\right)$

(11)The acceleration of the polished rod

$a=P \times \varepsilon_{p}$

\section{Kinematic analysis.}

(1) The polished rod load[3]

$$
W=\left.E f_{r} \frac{\partial u}{\partial x}\right|_{x=0}+f_{r} L\left(\rho_{r}-\rho_{y}\right)
$$

Where, the displacement of the any section $x$ of the sucker rod is $\mathrm{u}$; the elastic modulus of the pumping rod material is $E$; the sectional area of the sucker rod is $f_{r}$; the depth of the pump is $L$; the density of the sucker rod is $\rho_{r}$; the density of the well fluid is $\rho_{y}$.

(2) The net torque of brace axis

$$
M_{n}=\frac{N}{\omega}=\frac{V}{\omega} W-\frac{V_{1}}{\omega} Q \cos \beta-M_{A} \sin (\theta+\tau)
$$

Where, the velocity of the polished rod is $V$; the input power of the pumping unit is $N$; the polished rod load is $W$; the maximum balance torque is $M_{A}$; the balance weight is $Q$; the linear velocity of the balance weight of the oscillating guard bar is $V_{1}$; the crank balance phase angle is $\tau$, which associated with the pumping unit.

(3)Root mean square torque of brace axis[4]

$$
M_{e}=\sqrt{\frac{1}{2 \pi} \int_{0}^{2 \pi} M_{n}^{2} d \theta}
$$

(4) Average torque [4]

$$
M_{a}=\frac{1}{2 \pi} \int_{0}^{2 \pi} M_{n} d \theta
$$

(5) Load fluctuation coefficient[4]

$$
C L F=M_{e} / M_{a}
$$


(6) The rated power and effective power[4]

$$
N_{e}=\frac{M_{e} n}{9550 \eta}
$$

Where, the rate of pump is $n$; the efficiency of pumping unit is $\eta$.

\section{Application example}

The maximum stroke of the pumping unit is $3 \mathrm{~m}$; the rate of pump is 9 ; the pump setting is $889.8 \mathrm{~m}$; the submergence depth is $217.8 \mathrm{~m}$; the pump diameter is $70 \mathrm{~mm}$; the pumping rod diameter is $22 \mathrm{~mm}$; the tubing diameter is $63.5 \mathrm{~mm}$; moisture content is $93 \%$. Compared with the pumping unit, which is a conventional beam pumping unit. The net torque curve of the beam pumping unit and the swing crank-slide bar beam pumping unit is shown in Fig.3. The comparison of the main performance parameters of the conventional pumping unit and the designed pumping unit is shown inTable 1 .

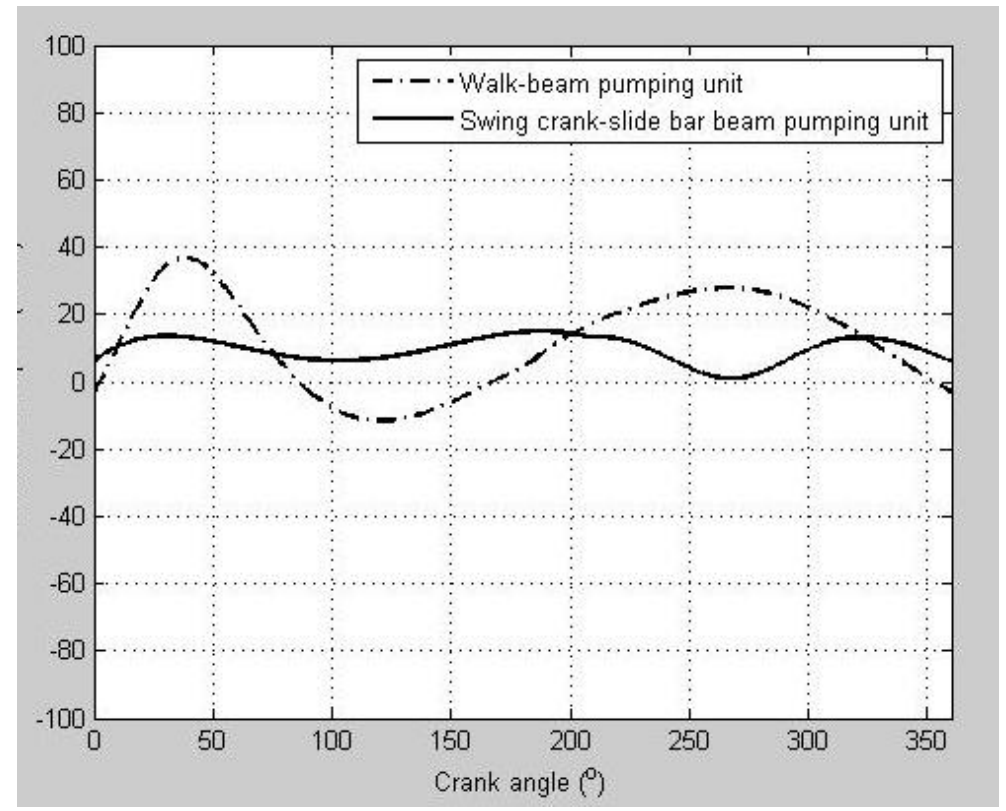

Fig. 3 The net torque curve of brace axis

Table 1 Comparison of main performance parameters of pumping unit

\begin{tabular}{ccccccccc}
\hline Pumping unit & $\begin{array}{c}\text { Stroke } \\
(\mathrm{m})\end{array}$ & $\begin{array}{c}\text { Upstroke } \\
\text { crank angle } \\
\left({ }^{\circ}\right)\end{array}$ & $\begin{array}{c}\text { Maximum } \\
\text { net torque } \\
(\mathrm{kN} . \mathrm{m})\end{array}$ & $\begin{array}{c}\text { Minimum } \\
\text { net torque } \\
(\mathrm{kN} . \mathrm{m})\end{array}$ & $\begin{array}{c}\text { Root mean } \\
\text { square torque } \\
(\mathrm{kN} . \mathrm{m})\end{array}$ & $\begin{array}{c}\text { Effective } \\
\text { output power } \\
(\mathrm{kw})\end{array}$ & CLF & $\begin{array}{c}\text { The power } \\
\text { saving rate }\end{array}$ \\
\hline $\begin{array}{c}\text { Conventional } \\
\text { pumping unit }\end{array}$ & 3 & 183.238 & 31.983 & -11.330 & 17.857 & 21.038 & 1.664 & 0 \\
$\begin{array}{c}\text { Novel } \\
\text { pumping unit }\end{array}$ & 3 & 222.811 & 12.19 & 5.305 & 9.777 & 11.519 & 1.009 & $45.247 \%$ \\
\hline
\end{tabular}

Compared with the chart 1 and table 3, we can see that the swing crank-slide bar beam pumping unit is better than the beam pumping unit in both the motion and dynamic parameters. The maximum net torque decreases from 31.983 to 12.19 , and decreases by $61.886 \%$; The minimum net torque increases from -11.330 to 5.305; the uneven loading factor CLF value decreases from 1.664 to 1.009; This greatly reduces the volatility of the net torque of brace axis and the effective output power. The wattful power saving rate reaches $45.247 \%$.

\section{Conclusions}

The net torque curve of brace axis of the novel swing crank-slide bar beam pumping unit is very smooth. The maximum value on net torque of brace axis is greatly reduced, and the minimum value 
effectively improved. The uneven loading factor CLF value approaches 1.The novel swing crank-slide bar beam pumping unit dramatically decreases the rated power and effective power, which indicates that the novel pumping unit has more notable energy conservation effect and better dynamic performance.

\section{Acknowledgements}

The authors would like to thank the Shaanxi Science and Technology Innovation Scheme (2015KTZDGY06-02), Key Problems of Industrial Science and technology of Shaanxi Province (2015GY110), (2016GY-185) and the Local Service Scheme of Shaanxi Province Education Department (15JF027) for financial support.

\section{References}

[1] T. Ren, Functional analysis of a new mode six-bar pumping unit, Mechanical Science and Technology.29(2010)1163-1167.

[2] B. Wan, Oil production equipment design and calculation, First edition,Petroleum Industry Press, Beijing, 1988.

[3] S. Dong, The design and computation of pumping unit and computer realization,Petroleum Industry Press, Beijing ,1994.

[4] W. Sun, The performance study of a novel pumping unit based on phase balance for cluster wells, Mechanical Science and Technology.33(2014)1869-1875. 